\title{
Social interest as an antidote to suicide: the cross-cultural applicability of an Adlerian solution
}

\author{
Marina Bluvshtein ${ }^{1}$, Filipp Filatov ${ }^{2 *}$, Makoto Kajino ${ }^{3}$, Antoine Jackson ${ }^{4}$ \\ 1 University of Chicago Center for Adlerian Practice and Scholarship at Adler \\ University,teaching Adlerian classes for Chicago, International Association of \\ Individual Psychology, and a faculty member of ICASSI; 60602, Chicago, USA \\ 2 Don State Technical University, Faculty "Psychology, Pedagogy and Defectology", \\ 344000 Rostov-on-Don, Russian Federation \\ 3 Takushoku University, Individual Psychology Association in Japan and Chimura \\ Clinic as a mental practitioner; 099-1123, Tokorogunoketocho, Tokyo, Japan \\ ${ }^{4}$ Adlerian counseling and Psychotherapy, 60602, Chicago, USA
}

\begin{abstract}
The article examines the applicability of an Adlerian view of suicide as sabotaged social interest to clients in a contemporary multicultural context. The approach examined here also focuses on common human factors in suicide and common useful approaches to working with people and communities affected by suicide. AdlerTs theory of suicide as sabotaged social interest was conceived and developed in the early 20th century, during a time of global political crises, economic chaos, and social unsettledness. Sadly, this makes this theory particularly applicable to the first two decades of the $21 \mathrm{st}$ century as well.
\end{abstract}

\section{Introduction}

The methods and rates of suicide vary by country. They are also affected by demographics and culture within a country, the mental health of those who attempt or commit suicide, the state of mental health care in a country, and sometimes by certain temporal factors, such as temporary economic distress or political instability. Sociodemographic variables (age, race, ethnicity, religious affiliation, socioeconomic status, gender, and sexual minority status) are some of the common factors addressed by contemporary clinical research on suicide; such research focuses primarily on suicide prediction, prevention, and intervention. Initially seen as a predominantly Western phenomenon, or one that primarily affects adolescents, Caucasian Protestant widowers

\footnotetext{
*Corresponding author: filatov_filipp@mail.ru
} 
or those in economic distress, suicide is a gradually growing mental health catastrophe that crosses both sociodemographic boundaries (class, race, and gender) and geopolitical borders.

\section{Adler's individual psychological view of suicide}

Heinz Ansbacher noted that Alfred Adler was the first to address suicide from the viewpoint of social psychiatry [1]. This includes considering the social backdrop of a case of suicide, as well as, in a uniquely Adlerian sense, viewing an individual's community (including the primary community of one's original family) as an active player in the development of suicidal tendencies, a major (albeit often without the awareness of all involved) part of the suicidal drama, and an equally active player in the individual's cure. Adler's first significant contribution was his paper "On suicide, with particular reference to suicide among young students", which was presented at the 1910 Vienna Psychoanalytic Society symposium. At this symposium, which was attended by Sigmund Freud, Josef Friedjung, Karl Molitor, David Ernst Oppenheimer, Rudolf Reitlert, J. Sadger, and Wilhelm Stekel, Adler argued that statistical data concerning "inadequate school equipment, errors in the educational system, and many other weak points in our civilization" could not provide "a single adequate, explanatory motive" or a fundamental cure for the "dynamic force that drives [one] out of life" [2, pp. 109-110].

This argument, made during the very early stages of the development of the theory of individual psychology, points to one of its fundamental tenets a kind of soft determinism that highlights the primacy of one's unique purposeful strategy in dealing with heredity and environment (in contrast to the conflict between heredity or environment alone) in understanding human nature. In 1937, Alexandra Adler noted, "You will find that the person who commits suicide is not as a rule, the person who get into the most dreary, ghastly, awful situation, such as losing a family member; he will not commit suicide. But you will find under conditions in which it seems to be much easier for the person, that person owing to some disappointment will either commit or try to commit suicide" [3, pp. 7-8].

After the 1910 symposium, Adler's attention to suicide peaked a few times during the time of economic depression and social pessimism in Austria that followed World War I, and again during the Great Depression that preceded the rise of the Nazi Party and eventually World War II. The other tenets in Adler's theory of suicide are that humans are socially embedded; that they are subjective and live in subjective realities built by fictions that they create and maintain; that they have the creative power to build, keep, or change these fictions; and that these fictions form a central theme in their goal-oriented movement toward a sense of perfection and completion. In his 1910 paper, Adler did not disregard situational factors, suicidal epidemics, gender, or ethnic differences. Yet, at that time and later, he saw these factors as secondary, emphasizing instead what people do when faced with the imminent demands of a "main tenet" and how socially useful these actions are. In 1921, Adler wrote that the dynamics of such movements is fueled by the expectation of new events which imply for the neurotic new struggles, new dangers of a setback and by the neurotic's choice to proceed "immediately to intensive protective measures, the most extreme of which is suicide" [4].

Adler considered control and perfectionism significant; those who attempt suicide are often severely discouraged, fear they will never reach their own self-imposed standard of perfection, and lack the courage to risk imperfection. Their sense of belonging bleeds from self-inflicted wounds. They come to believe that staging a flawless death is the only way they can restore their sense of control. This is how 
suicide becomes a solution for a person "who in the face of an urgent problem has arrived at the end of his limited Social Interest" [1, p. 323] and therefore lets go of the last string tying them to humankind. This leap (or escape, as Adler frequently referred to it) away from social interest is active, often dramatic, and always relational; a paradoxical communication lies at its heart [2].

The paradox of a suicidal dialogue with the world can be seen on two levels: the suicide hurts others by injuring themselves and also benefits from their own actions without facing the consequences $[1,2]$. The very paradoxical nature of suicide should be seen in how a person might imagine they will achieve literal emotional completeness through self-destruction. The paradox imbedded in these ideas, alarming to a healthy person, seems to be guide those with "a heightened feeling of superiority" who are well on their way "to be master of life and death" [2, p. 151]. In 1974, Neuringer noted the "lessening of creative flexibility and narrowing of the conceptual range" (p. 61) in those with suicidal ideations. That cognitive rigidity may heighten the subjective urgency in such a personTs perception of the challenges to be solved.

\section{Culture and suicide}

For more than a century, suicide has been a global mental health catastrophe slowly migrating from country to country, with peaks at what seem to be particularly suicidogenic times and in what are reported to be suicide-prone demographic groups. The World Health Organization includes suicide among the three leading causes of death among 15- to 29-year-olds worldwide; it accounts for $50 \%$ of menTs violent deaths and $71 \%$ of women's (WHO, 2014). In the United States, suicidal ideations and suicidal attempts are reported to be higher among Latino and American Indian adolescents, younger urban African-American males, older Protestant widowers, active duty military personnel, immigrants from areas with high levels of violence, and college students in general and LGBTQ students in particular (Akkaya-Kalayci et al., 2015; Barlow et al., 2010; Chiurliza, Michaels, \& Joiner, 2016; Jobes, 2013; Malmin, 2013; Saito, Klibert, \& Langhinrichsen-Rohling, 2013; Silva et al., 2015; Wadsworth, Kubrin, $\&$ Herting, 2014) [8]. Young adults have the highest rate of suicidal attempts; according to the Center for Disease Control and Prevention (CDC), young people aged 15 to 24 attempt 100 to 200 suicides for each completed suicide (CDC, 2011). Females have a generally higher rate of suicidal ideation and a higher incidence of suicide attempts but a lower rate of completed suicides (APA, 2010).

These statistics must be approached with caution; underreporting and misinterpretations are common, especially in statistics about suicide among people of color and those with low socioeconomic status (Kapusta et al., 2011; Rockett et al., 2010). On the other hand, suicides committed by African-American and Latino youth may not always be reported as suicides, especially murder-suicides, victim-precipitated suicides, and suicides in the prison system [8]. Outside the United States, solid studies on suicide are mostly limited to the countries where suicide is more common, such as Japan and South Korea (Flaskerud, 2014; Picone, 2012), and European countries that allow or are considering the legalization of euthanasia and physician-assisted suicide (Radbruch et al., 2016; Stoltz et al., 2015). Finally, the lack of valid cross-cultural and ethical consensus on a definition of suicide, along with the myriad of ways potentially suicidal behavior is "perceived, labeled, and tolerated" in different cultures [10, p. 16], present unique challenges for anyone seeking cultural meanings of and culturally appropriate cures for suicide. Aside from the expected limitations of any statistics, the major criticism of contemporary studies of suicidality has been their excessive focus on structural/demographic variables rather than on the symbolic meaning of suicide or on 
an individual's values as predictors of social (and therefore individual) attitudes toward suicide and tolerance of it (Stack \& Krosowa, 2016).

Culture, in a broad sense, is considered a factor in most studies on suicide. It is usually seen as a risk factor that scripts self-destructive behaviors. For example, in industrialized countries, a man's suicide may be seen as masculine and less deviant than a woman's suicide (Canetto, 2010). Suicide rates among American Indian youth may be attributed to their historic and continuing chronic cultural oppression (Barlow et al., 2010), and increasing cultural self-expressionism and decreasing cultural survivalism may lead to greater tolerance for suicide in some countries (Stack \& Krosowa, 2016). Adler's theory of individual psychology is uniquely equipped to support an examination of cultural scripts related to life and death, as well as the unique individual choices that make meaning of these cultural scripts. Applied to the issue of suicide, this theory might make it possible to move beyond risk factors and into the realm of healing.

\section{A case of three cultures}

The relatively low (compared to other minority groups) reported suicidality among African Americans in the United States may seem surprising. African Americans (particularly the descendants of slaves) are one of the most disconnected cultural groups in the United States. When not used as backdrops for political rallies or as favorable employee statistics, African Americans are often dehumanized, despised, and unconsciously feared. In the media, they are often depicted as wild and untamable, people who must be incarcerated and rehabilitated by the justice system. The criminal justice system, meanwhile, has had little success addressing the overrepresentation of African Americans in prison populations and its own unpreparedness to work with this population (McCarter, 2009; Richardson, Brown, \& Van Brakle, 2013). African Americans have the lowest access to basic resources of any group in the United States. Faced with all these social stressors, in addition to any personal stress, it seems that African Americans have every plausible reason for having the highest rates of suicide. However, the available statistics tell us otherwise. Why?

African Americans rarely discuss suicidal thoughts or even suicidal behavior from an individual perspective, but the community as a whole does speak about aspects of cultural suicide and engage in culturally suicidal behavior. Cultural suicide is a deeply rooted hatred for one's own cultural identity that leads to purposely self-destructive messaging and behavior toward members of one's own culture. The cultural identity of the African people who were kidnapped and forced into slavery almost 500 years ago was deliberately destroyed by the people who placed them in captivity. Their original African human identity was stripped away, and they were left with the reflection of an American husk that they were forced to accept as their new identity. However, not only did they dislike this reflection; they often hated it. The devaluing of black life by black people can be traced back to the "doll test" research done by Kenneth and Mamie Clark during the 1954 Supreme Court case of Brown v. Board of Education. Replicated multiple times over the past 70-plus years, this experiment still reflects black peopleTs (in this case, black childrenTs) significantly lower valuing of black life (Zirkel, 2005 [19]). Black-on-Black violent crime is the number one cause of African American deaths in the United States; this statistic is even more significant for black men (FBI, 2014; U.S. Department of Justice, 2007). Many movies from the late 70s through the 90s strongly suggest that African Americans (especially men) aspire to be pimps, drug dealers, gangsters, and most certainly murderers and that this behavior occurs primarily within their own communities. Movies such as a New Jack City, Boyz-N-Da-Hood, and a Menace II Society so encouraged Black-on-Black crime that, in many states, African 
Americans committed violent crimes or even murdered other African Americans in the parking lots of movie theaters immediately after watching these movies.

In a broader social and cultural context, in evolutionary terms, African-American culture is killing itself. African-American culture decreases at the hands of members of that very culture with each such crime. This is cultural suicide.

The cultural aspects of suicide in Japan are also worth discussion. In Japan, the suicide rate per 100,000 persons $(18.9 \%$ in 2015$)$ is much higher than that in the United States (12.4\% in 2014) (National Police Agency of Japan, 2016). Suicides in Japan also employ very different methods from those in the United States. Because Japanese citizen are not allowed to own firearms, the most common methods of suicide are jumping in front of a train, inhaling gas, and overdosing [12]. Japanese culture holds comparatively positive views of taking one's own life. It is generally much more tolerable than in the United States, and it is often expected or even prescribed in certain social strata. During Japan's Warring States period, the samurai warriors had a ritual: In seppuku or hara-kiri, they killed themselves by cutting their own abdomens. This was done to avoid providing information to the enemy because of their great loyalty to their lord. Even their enemies understood and respected the samuraisT need to keep face and honored the ritual of hara-kiri.

In Japan, suicide is seen as a morally responsible action and as a way of taking responsibility for perceived failures and the problems caused by these failures. For example, a person who is failing in business and causing their company large losses might feel guilty enough to choose death without any hesitation [12]. The Japanese agriculture minister Toshikatsu Matsuoka, who hanged himself in 2005, left eight notes about taking responsibility for his problems and failures and the inconvenience they had caused (Beam, 2007).

The high suicide rate in Japan must be considered in light of the tendency, common in Japan, to have an external locus of control and an internal locus of responsibility (ECIR). When experiencing hardship, the Japanese often say shiganai; this suggests that the situation cannot be helped or is beyond their power. This acknowledgment of external control is accompanied by a deep sense of internal personal responsibility for specific situations. This requires inner strength and resolve in the face of out-of-control situations and often leads to suicide as a way to accept responsibility for perceived failures. Accepting responsibility and acting on that acceptance (in the form of seppuku or hara-kiri) is perceived as a way to avoid shame.

Interestingly, a similar phenomenon is found in another place far from Japan. In the sub-Saharan African language of Yoruba (per Atilola \& Ayinde, 2015), there is no single word for suicide. However, a phrase used to describe suicide is literally translated as "he/she acted like a man"; this reflects a masculine ethos that requires a person to be man enough to face death rather than shame, dishonor, and indignity (p. 410)

In pre-Soviet Russia, the Soviet Union, and post-Soviet Russia, self-destructive behaviors have peaked during the cultural, political, and economic crises that marked breakups of social interaction, compromised the basic values of collective life, and widened the gap in systemic connections. Because Russia's political crises in the 20th century have frequently led to more (rather than less) economic and social stress, overall suicide rates did not decrease significantly between these crises. Suicide rates in Russia increased in the second part of the 20th century, but the types of suicides shifted, as did the population groups most affected by suicide.

The beginning of the 20th century saw numerous cases of self-harm, self-torture, and suicide (committed not by individuals but by entire families) based on religious beliefs. These cases, frightening due to their irrational self-destructiveness, were 
observed mainly among Russian schismatics in remote settlements. In these cases, selfimmolation and self-burying were often seen as ways to atone for sin.

In addition to age, gender, period, and the cohort effect (Bukin, 2019; Dvoryanchikov et al., 2014; Jukkala et al., 2017), two types of suicide should be considered culturally specific to Russia. In the Soviet Union, one type of suicide was a heroic death. During World War II, soldiers sometimes deliberately sought death, usually killing some enemies along with themselves, often for the higher ideals. They became role models and were immortalized by Soviet propaganda. Such soldiers might fly a burning plane into an enemy military formation or burn themselves alive in a house surrounded by the enemy rather than surrender. They valued devotion to the homeland over their own lives. During and following the collapse of the Soviet Union, a second type of suicide emerged. Many viewed the collapse of the Soviet Union as an apocalypse [3], the end of the great utopian project, the end of all previously held and cherished values. Some who held this view committed suicide; a prime example is the poet Julia Drunina. In her youth, she repeatedly demonstrated heroism during the war. She was a well-known poet. However, she committed suicide on November 21, 1991, unable to survive the collapse of the social ideals to which she had devoted her life.

Many cases of Russian suicide illustrate the value of collective life exceeding that of individual life, which is consciously sacrificed or destroyed when the familiar social context and the values of that context are lost. Added to that, in Russia today, a growing number of groups at high risk of suicide parallel high-risk groups in other countries, such as youth from culturally disadvantaged populations and people who live in economically depressed areas (Slobodskaya \& Semenova, 2016).

\section{The advantage of Adlerian views on suicide to a culturally meaningful clinical practice}

Several ideas from the theory of Individual Psychology related to suicidality present a clear advantage over other theories for working with systems affected by suicide and for addressing the many indivisible relational facets of suicidal ideation, contemplation, and completion. Through the lenses of other theories, a suicide could be seen as one a distinct individual cutting ties with humanity, rather than relational, a systemic phenomenon.

According to cognitive-behavioral therapeutic approaches, hopelessness and poor coping skills are primarily responsible for suicidal ideation and suicidal attempts (Beck, 2010; Wittouck et al., 2014). When negative life events and certain demographic characteristics are added to the mix, these two ingredients elevate an individual's risk of suicide, either immediately or, if these issues are poorly controlled and chronic, over their lifetime. Although these two constructs are central to many suicidal screening tools, such as the Beck Hopelessness Scale (BHS), they are not culturally universal. In some immigrant communities, feeling of hopelessness may not be a top predictor of suicide; they may give way to other feelings, such as worthlessness, as is the case with Somali immigrants in the United States (Schoener, 2009 [17]). The construct of hopelessness as used by Beck and in follow-up research is unlikely to carry the historical communal load, a central ingredient to the communal hopelessness expressed in suicidal ideations by African-American descendants of slaves or victims of the Holocaust. It also fails to account for unique, culturally prescribed response styles as a contextual factor in these populations. Adler, on the other hand, notes that suicidal ideators demonstrate, among other characteristics, a strong reduction of the worth of life (1021, p. 209). On a social level, especially for historically oppressed groups, 
devaluation of one's own life should be considered in connection with and understood to be impacted by institutional invalidation.

The interpersonal theory of suicide seems to be closer to Adler's individual psychology in its holistic understanding of suicide. This theory posits that the chances of suicide increase when two elements are simultaneously present: a thwarted sense of belonging and a perception of oneself as a burden (Chiurliza, Michaels, \& Joiner, 2016; Van Orden et al., 2010). According to this theory, the addition of hopelessness about the two states above and about one's ability to commit suicide, along with habituation to suicidal experiences and increased pain tolerance due to previous repeated attempts, significantly increase the probability of suicide. Still, the main practical uses of the theory, as evidenced in the available literature, seem to involve identifying the risks, so treatment remains primarily symptom-based.

In general research, deficits in family and communal functioning are implicated in increased suicide risk, and the presence of family support and of strong cultural and communal identification is frequently indicated as a protective factor for most sociodemographic groups, especially minority ones $[6,10]$. However, only a few studies have investigated a community-based disincentive for suicidal behavior (Oquendo et al., 2005); communal values that actively proscribe suicide as a crime against the community [5]; or communal morals that in some cultures, may encourage increased acceptance of suicide as a kind of self-expression (Stack \& Krosowa, 2016). Similarly, in non-Adlerian research, with a few exceptions (such as the discussion of a suicide of resolve in Kitanaka, 2008 [14]), insufficient attention has been given to the unique individual or cultural meaning of suicide (Lester, 2011/2012). The latter, however, is not always seen as the best way to view the psychodynamics of suicide, and Borum [8] argues that the Afrocentric approach that views individuals as permanently extended into their communities and communities as an extended self is the only valid approach to understanding the African-American view of suicide.

Adler included suicide in his list of failures that are lacking in social interest [2]. Adler's view that suicide lies on a universal continuum of predispositions to solve life challenges by removing oneself from life versus joining in with life and solving life challenges in a useful way, can have social and clinical value beyond a person's declared or assumed cultural affiliation [6]. Cultural ethos, prescriptions, and proscriptions become added factors, shaping an individual's artfully nuanced strategy development and a pattern of actual movement in life.

According to Adler, the essence of a suicidal attempt is an attempt to avoid the risks and frustrations of dealing with communal feelings, with the demands of communal life, and with the individual responsibility for communal survival. While it applies at all times, this kind of moving backward in which every type of useful activity is prevented and the patient remains victorious over the normal social obligations [2] can be especially prominent in times of communal crisis, whether local or global. This is true, not because of the environmental risk factor of the disintegration of communal fabric, but because, in these times of crisis, the call for communal feelings may be more pronounced and unavoidable, and those who lack this communal feeling might respond to such a call by becoming permanently unavailable.

Adler's theory of individual psychology offers a non-deterministic, non-reductionist, teleological view of and approach to suicide that is cross-culturally valid. An Adlerian focus on the purpose (rather than the cause) of suicide is a key to a socially useful crosscultural application of Adlerian psychology that is not tied to restrictive, mono-cultural causative variables (environmental factors and biological markers). This approach is more congruent with the multicultural reality of the 21 st century not many but one humanity than other approaches [15]. Adler's focus on an impaired sense of belonging 
and people's strategies for dealing with that impairment (which is a core in any psychopathology) is a perfect fit for the cross-cultural reality that we all experience, in which culture shapes our creative strategic response to perceived life challenges on individual and communal levels. Whether one's current feeling of not belonging is linked to a stored historical communal trauma or to a recent event, an Adlerian focus on the meaning of the felt trauma for one's today and the purpose of that feeling for one's tomorrow brings hope to what may be seen as ultimate and irreparable hopelessness.

\section{References}

1. H.L. Ansbacher, Suicide as communication: Adler's concept and current applications. Journal of Individual Psychology, 25(2), 174- 180 (1969)

2. H.L. Ansbacher, R.R. Ansbacher, The Individual Psychology of Alfred Adler: A systematic presentation in selection from his writings. (Harper \& Row, Publishers, New York, 1964)

3. A. Adler, Drunkenness and suicide. Journal of Individual Psychology, 71(1), 413 (2015)

4. A. Adler, The Neurotic Constitution. (Moffat, Yard and Company, New York, 1921)

5. O. Atilola, O. Ayinde, The suicide of Șàngó through the prism of Integrated Motivational - Volitional model of suicide: implications for culturally sensitive public education among the Yorùbá. Mental Health, Religion \& Culture, 18(5), 408-417 (2015)

6. A. Barlow, B.C. Mullany, N. Neault, Y. Davis, T. Billy, R. Hastings, Coho- V. Mescal, K. Lake, J. Powers, E. Clouse, R. Reid, J.T. Walkup, The Journal of the National Center, 17(1), 1-24 (2010)

7. A.T. Beck, Community-based cognitive therapy for suicide attempters (National Institute of Health, 2010)

8. V. Borum, Social Work in Public Health, 29, 656-670 (2014) DOI: 10.1080/19371918.2013.776339

9. P. Friedman, Discussions of the Vienna Psychoanalytic Society, 1910 "On Suicide, with particular reference to suicide among young students". (International Universities Press, New York, 1967)

10. D.B. Goldston, S.D. Molock, L.B. Whitbeck, J.L. Murakami, L.H. Zayas, G.C. Nagayama, American Psychologist, 63(1), 14 - 31 (2008)

11. L.A. Johnson-Migalski, The Journal of Individual Psychology, 67(1), 31-40 (2011)

12. M. Kajino, Journal of Individual Psychology, 68(4), 337-350 (2012)

13. M. Kajino, The Development and Expansion of Individual Psychology in Japan (Adler Graduate School, MN, 2014)

14. J. Kitanaka, Cultural Medical Psychiatry, 32, 152-176 (2008) DOI 10.1007/s11013-008-9087-1

15. G.J. Mozdzierz, The Journal of Individual Psychology, 54(1), 1-3 (1998)

16. C. Neuringer, Journal of Individual Psychology, 30 (1), 59-64 (1974)

17. G.R. Schoener, A high-risk situation: The suicidal client. Minnesota Psychologist (Spring, 2009)

18. K. Slade, R.C. Edelman, The Journal of Crisis Intervention and Suicide Prevention, 35(2), 82-89 (2014)

19. S. Zirkel, Urban Review, 37(2), 107-126 (2005) 